\title{
NEURAL NETWORK CONTROL DESIGN CONSIDERATIONS FOR THE ACTIVE DAMPING OF A SMART BEAM
}

\author{
Piotr Cupią \\ AGH University of Science and Technology, Kraków, Poland; e-mail: pcupial@agh.edu.pl \\ ŁUKASZ ŁACNY \\ Cracow University of Technology, Mechanical Faculty, Kraków, Poland; e-mail: llacny@pk.edu.pl
}

\begin{abstract}
In this study, possible options for the active damping of a smart beam with piezoelectric patches using neural network control algorithm, are presented. The algorithms used for the control are Neural Direct Inverse and Feedback Linearisation (NARMA-L2). Additionally, several possible modifications used for the purpose of improving the control, such as different values of control gain or sampling time of the training data, as well as step-wise control are tested.
\end{abstract}

Keywords: neural network control, smart structures, vibration damping

\section{Introduction}

In the recent years, the interest in the so-called "smart structures" and their application in real-life systems has greatly increased. The advantages of such structures - to monitor even subtle changes in their environment and actively respond and react to them - cannot be overlooked. Numerous possible uses of this technology have already been developed and discussed, e.g., structural health monitoring, active vibration and noise damping, energy harvesting (Choi, 2002; Crawley and Luis, 1987; Cupiał, 2008; Preumont, 1997; Tylikowski, 1993).

Neural networks, on the other hand, have been successfully used mostly for the purpose of identification and pattern recognition (Flasiński, 2011; Rutkowski, 2009), but also to some extent in the control of both linear and non-linear systems (Hagan and Demuth, 1999; Narendra et al., 1990; Nøorgaard et al., 2003; Omidvar and Elliot, 1997; Passino, 2005). The possibility of combining smart materials with neural network control could provide us with systems responding to external excitation and adjusting to it accordingly.

This paper focuses on the possibility of using such a combination in order to improve the damping performance of an oscillating system, i.e., a clamped beam with piezoelectric patches. The option of using neural networks for the damping of similar "smart structures" has already been attempted by several authors. In these studies, the neural network was either used to emulate an existing control method (Smyser and Chandrashekhara, 1997) and to adjust the parameters of such a method, e.g., find the optimal values for $K_{P}, K_{I}$ and $K_{D}$ of an PID controller (Qiu et al., 2012) or the network was of a recurrent type (Valoor et al., 2001).

Each of these methods is correct from the analytical point of view and some have been proved (both numerically and by means of an experiment) to reduce the vibration of an oscillating object efficiently. However, it would be preferable if the neural control method were not derived from an existing control algorithm and would be derived from the dynamics of the systems alone. Additionally, it is necessary that the artificial neural network used for the control is both simple to implement and use.

In this paper, the damping is performed by methods that utilize neural networks of the feedforward type, while the training is based only on the samples from the existing model 
(without assuming any pre-existing control). In addition to that, several adjustments to control are considered for the purpose of increasing the performance even further.

\section{Model of the smart beam}

In this paper, an effort is made to present possible means of damping the vibration of a smart beam using artificial neural networks. The "smart beam" in question is a clamped-free beam with two piezoelectric patches attached next to the clamping end, as shown in the simplified model (Fig. 1). The patches operate as a collocated pair - one acting as a sensor while the other one as an actuator (Preumont, 1997). It is assumed in the model presented in this work that the external excitation is applied in the form of an impulse at the free end of the beam, perpendicular to the beam. This assumption allows for the model to be simplified by considering only the transverse vibration of the beam, while it also ensures that the higher vibration modes are excited as well.

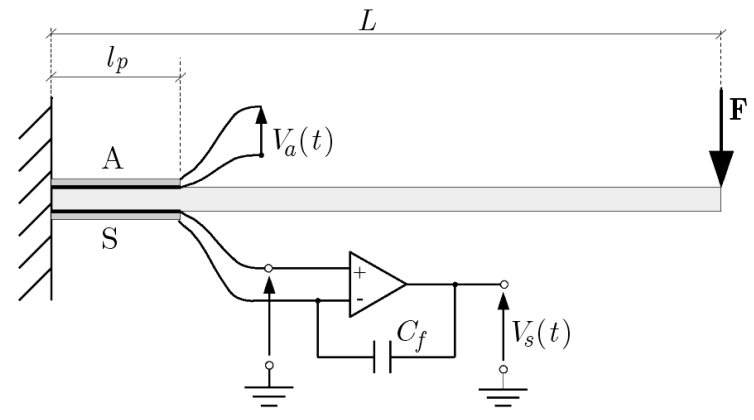

Fig. 1. Simplified model of a smart beam with piezoelectric patches (A - actuator, $\mathrm{S}$ - sensor) and a charge amplifier $\left(C_{f}\right)$

The dimensions and material properties of the beam considered in the analysis are given as follows

$$
\begin{array}{ll}
L=1.5 \cdot 10^{-1} \mathrm{~m} & - \text { length of beam } \\
h=1.5 \cdot 10^{-3} \mathrm{~m} & - \text { height of beam } \\
b=10^{-2} \mathrm{~m} & - \text { thickness of beam } \\
\rho=7.9 \cdot 10^{3} \mathrm{~kg} / \mathrm{m}^{3} & - \text { beam material density } \\
E=2.1 \cdot 10^{11} \mathrm{~N} / \mathrm{m}^{2} & - \text { Young's modulus }
\end{array}
$$

Using these parameters and one of the available methods (Rayleigh-Ritz, Galerkin, etc., see Nixioł, 1996) the natural frequencies of the beam can be obtained.

For the additional simplification of the model, the effect of the piezoelectric patches can be defined using the following equations (Preumont, 1997)

$$
M(t)=c V_{a}(t) \quad V_{s}(t)=s\left[\left.\frac{\partial w(x, t)}{\partial x}\right|_{x=x_{b}}-\left.\frac{\partial w(x, t)}{\partial x}\right|_{x=x_{a}}\right]
$$

Equation $(2.1)_{1}$ relates the moments at both ends of the piezo-actuator with coordinates $x_{a}$, $x_{b}$ to the applied voltage. The variable $x$ in this equation defines the position on the beam, while the parameter $w$ is the deflection of the beam at a given point and time. Equation $(2.1)_{2}$, on the other hand, gives the relation between the angle of rotation of both ends of the piezo-sensor and the voltage generated in the sensor. Each of these equations contains additional parameters $c$ 
and $s$ which are dependent on the material properties and the dimensions of the patches. These parameters can be calculated using the following formulas (Preumont, 1997)

$$
c=\frac{E_{p}\left|d_{31}\right| b_{p}\left(h+h_{p}\right)}{2} \quad s=\frac{E_{p}\left|d_{31}\right| b_{p}\left(h+h_{p}\right)}{2} \frac{1}{C_{f}}
$$

in which $h$ is the height of the beam, $E_{p}$ and $d_{31}$ are Young's modulus and the piezoelectric constant of the patches, while $b_{p}$ and $h_{p}$ is the thickness and height of the patches, respectively. $C_{f}$ appearing in Eq. $(2.2)_{2}$ is the capacitance of the charge amplifier. For the purpose of this analysis, these parameters are chosen as follows

$$
\begin{array}{lll}
E_{p}=50 \cdot 10^{9} \mathrm{~N} / \mathrm{m}^{2} & d_{31}=-150 \cdot 10^{-12} \mathrm{C} / \mathrm{V} & b_{p}=10^{-2} \mathrm{~m} \\
h_{p}=1.5 \cdot 10^{-3} \mathrm{~m} & l_{p}=2 \cdot 10^{-2} \mathrm{~m} & C_{f}=10^{-7} \mathrm{C}
\end{array}
$$

Additional information about piezoelectric materials and their application both as sensors and actuators can be found in the literature (Crawley and Luis, 1987; Cupiał, 2008; Preumont, 1997).

\section{Artificial neural network control}

\subsection{Control considerations}

Before an attempt at any actual control by neural networks can be made, several important aspects of this control need to be considered. This step is essential, due to the complex nature of neural networks the behaviour of which may be influenced by many different factors. Below, the listing of the most relevant factors is presented along with a short explanation for each one of these decisions as well as for the choices made during the control design:

- type of control - although the model considered in this paper could be regarded as MIMO, the control becomes much simpler if it is considered as SISO, which is especially true when trying to train the appropriate network for control. The input in this case is the voltage applied to the actuator. As for the output - either the voltage measured by the patch sensor or the displacement of the beam free end seem appropriate. In fact both of these have been tested for this paper and the results are similar;

- neural controller type - even though there are several types of neural controllers available in the literature (Hagan and Demuth, 1999; Korbicz et al., 1994; Nøorgaard et al., 2003), not all of them can be efficient when applied to the model in question. Although the control using one of the direct method controllers (Direct Inverse and Feedback Linearisation) proved possible, albeit with slight modifications, an attempt to damp the beam by means of Predictive Control was found to be inefficient;

- neural network design - specifically: number of layers, number of regressors, size of layers (especially the hidden ones) and the type of activation functions. All of these parameters influence the way the network behaves, and how well it can perform;

- training data - the data collected from the model (either experimental or generated numerically) needs to be adequate for the training of the network. First of all, the training samples should contain, if possible, the whole range within which the control will be performed. This, in turn, requires the input (excitation) to be chosen correctly, either as a random- or as a chirp function. Additionally, a decision needs to be made regarding the sampling time and the amount of samples - in both of these cases, an optimal value must be found or the network may not operate as intended; 
- training method and performance - in the case of dynamical neural networks the preferable method of training is the so-called Lavenberg-Margquardt method (although not always, e.g, with Model Reference Control). As for the training performance (in this case calculated as a mean square error between the target- and the actual output value), it can be shown that problems may occur both when it is too low (inefficient control) or too high (super-efficient control, but with the control effort reaching unrealisable values). Similar to the training data generation, an optimum needs to be found.

The two neural control methods used for damping of the smart beam are: the Direct Inverseand Feedback Linearisation (NARMA-L2) Control. In the former method, an assumption is made that the neural network can be trained to emulate the behaviour of the inverse of the system. By supplying the already trained controller with a desired reference value, the network can calculate the control force needed to reach that reference. The equation describing the inverse function is given as follows

$$
\widehat{u}(k)=\widehat{f}^{-1}\left(y_{r}(k+1), y(k), \ldots, y(k-n), u(k-1), \ldots, u(k-m)\right)
$$

with $\widehat{u}(k)$ representing the control force, $y_{r}(k+1)$ - the reference value, $u(k), \ldots, u(k-m)-$ control regressors and $y(k), \ldots, y(k-n)$ - output regressors.

The second control method (NARMA-L2) defines the control value using the linearised equation

$$
\widehat{u}(k)=\frac{y_{r}(k)-f(k)}{g(k)}
$$

where $y_{r}(t)$ is the reference signal, while $f(k)$ and $g(k)$ are non-linear functions of the control and output regressors

$$
\begin{aligned}
& f(k)=f(y(k), \ldots, y(k-n+1), u(k-1), \ldots, u(k-m)) \\
& g(k)=g(y(k), \ldots, y(k-n+1), u(k-1), \ldots, u(k-m))
\end{aligned}
$$

Both non-linear functions are trained using separate neural networks using the data generated (or obtained) from the model.

A more detailed description of these NN control methods can be found in the papers by Nøorgaard et al. (2003), Hagan and Demuth (1999), Medsker and Jain (1999).

\subsection{Neural network control}

The data used for the training of the neural controllers are generated using the available smart beam model, described in Section 2. The voltage applied to the piezo-actuator during the data generation is limited to the range $[-250 \mathrm{~V}, 250 \mathrm{~V}]$. It is necessary to impose these limits to ensure that a feasible value of voltage is applied during the simulation and eventual control. In the simulation studies an additional saturation block has therefore been added.

Although the two control methods used in this paper differ in terms of the network structure, the results of control are similar with only minor differences noticeable. Therefore, only the results of one of these methods are shown in the following tests. Apart from the active damping, additional passive modal damping of value $\zeta=0.001$ for each mode is present in the model. Unless stated otherwise, the default values used for the tests discussed below are as follows: control gain $g=0.01$, sampling time $\Delta t=0.002 \mathrm{~s}$. 


\subsubsection{NN control - relation to training data}

Figure 2 shows how the sampling time of the data used for the training of the neural controller influences its behaviour. It needs to be noted that in each case the network architecture as well as the number of training iterations and the desired training performance is the same. It is imperative that the sampling time $\Delta t$ is small enough to include the dynamics of the controlled system with enough precision. However, as can be seen in Fig. 2, the higher the sampling frequency, the faster the network tries to regulate the system, which leads to a much higher control voltage. If not for the saturation block, this would have caused the voltage applied to the piezo-actuator exceed the values imposed during the design. On the other hand (Fig. 2c), if the sampling time $\Delta t$ is too small, the active damping is less effective. Therefore, it can be seen that the control performance of the neural network controller is related to the training data chosen, and it should be considered beforehand.

(a)

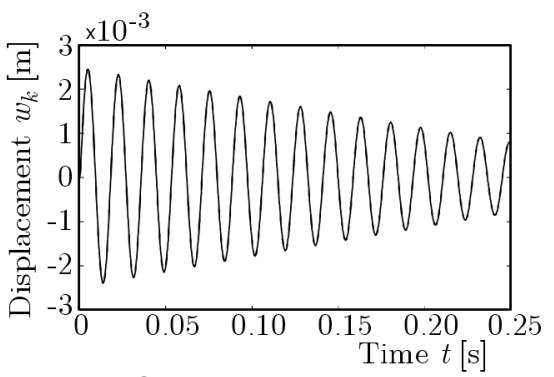

(b)

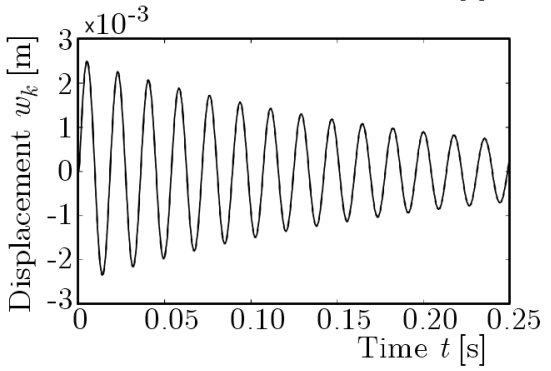

(c)

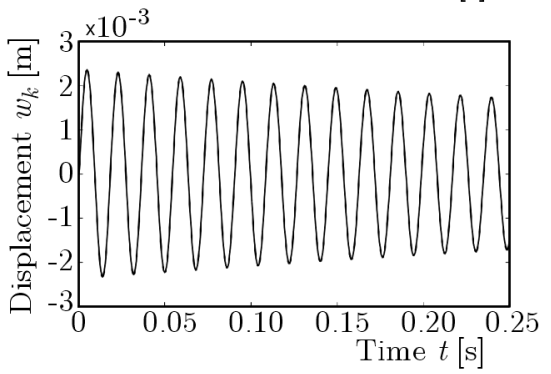

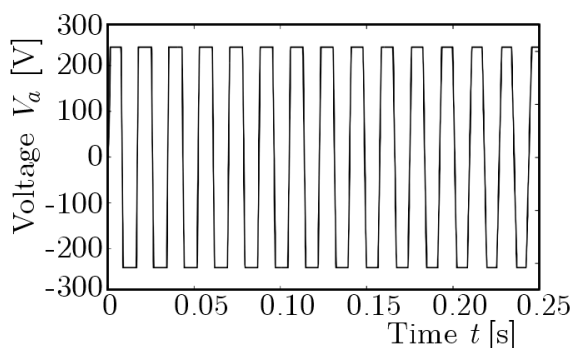
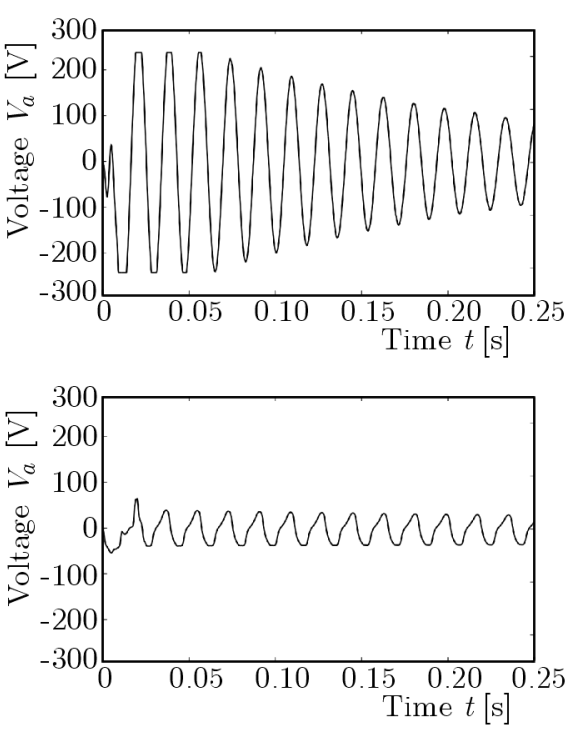

Fig. 2. Displacement of the free end of the beam $w_{k}$ and control voltage $V_{a}$ for different training data sampling times: (a) $\Delta t=0.001 \mathrm{~s}$, (b) $\Delta t=0.002 \mathrm{~s}$, (c) $\Delta t=0.005 \mathrm{~s}$

\subsubsection{NN control - relation to gain value}

The results of using three different values of the control gain are shown in Fig. 3. The efficiency of damping can be improved by increasing the gain, but obviously at a cost of the increased voltage.

\subsection{3. $N N$ Control with the step-wise change of target value}

As neural network controllers show a tendency to choose the best possible (mostly highest) control value to reach the desired point, it may be possible to improve their behaviour by setting 
(a)

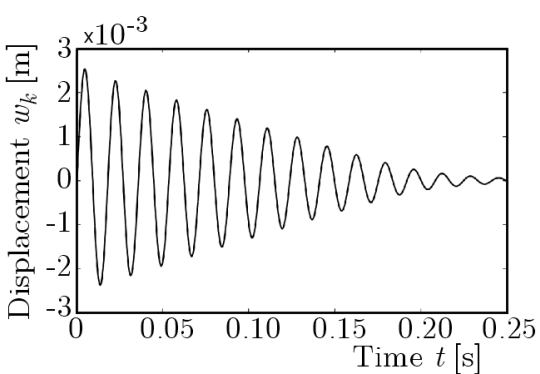

(b)

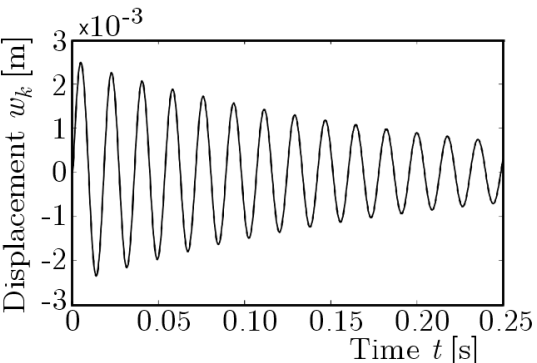

(c)

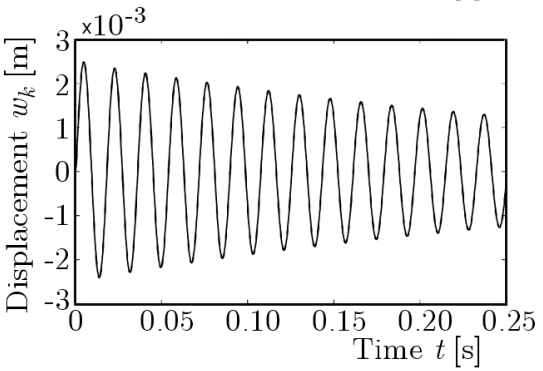

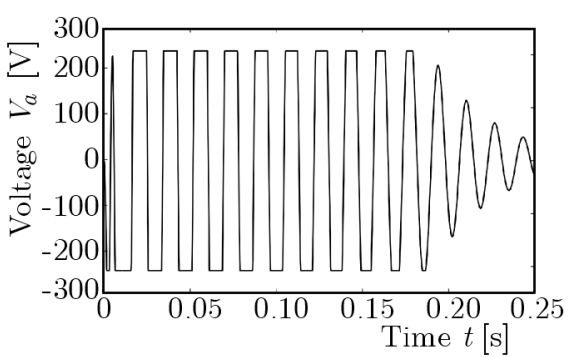
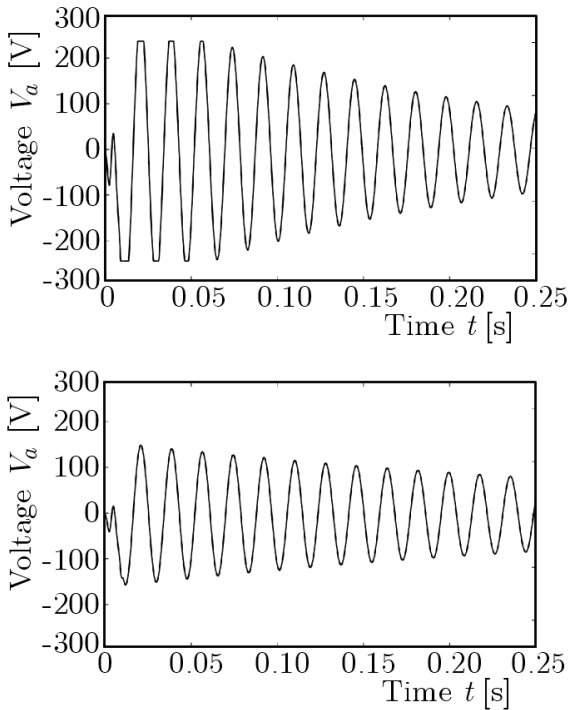

Fig. 3. Displacement of the free end of the beam $w_{k}$ and control voltage $V_{a}$ for different values of gain: (a) $g=0.1$, (b) $g=0.01$, (c) $g=0.005$

interim goals for the controller, leading to the final goal. In this case, an additional step-wise change block is added, which in each step replaces the setpoint value $t$ of the displacement of the free end of the beam by a value that lies between the actual value $y$ and the setpoint. This new target value can, in general, be found using the following equation

$$
\widehat{t}=y(1-d)+t d
$$

where $d \in[0,1]$ is the descent ratio. In our case this is simplified since $t=0$. If $d=1$, the control is changed to default (as if no modification were introduced). On the other hand, when $d=0$, no control can be performed as the actual output $y$ is the same as the modified target output $\hat{t}$. By adjusting the value of ratio $d$, the controller can be forced to reach the target more slowly and, in turn, to decrease the required control voltage. In Fig. 4, it can be seen that for $d=0.1$ the control values are visibly lower with no noticeable difference in the control performance.

\subsubsection{NN Control with stepwise control (higher modes)}

The control of several modes (in this case the 1st and 2nd one) can be seen in Fig. 5, respectively, with the standard- and stepwise control. The results are similar to those discussed before. It should be noted that the control in this case is performed using the controller trained only for the first mode and then modified to operate for both modes. In this approach, the controller sampling time remains unchanged. On the other hand, the system sampling time is made shorter by dividing the controller sampling time by a natural number $r$. This means that instead of using the output regressor $y(k-n)$, the regressor $y(k-r \cdot n)$ is used. The same rule applies to the input regressors. However, one should be careful when using this modification. 
(a)

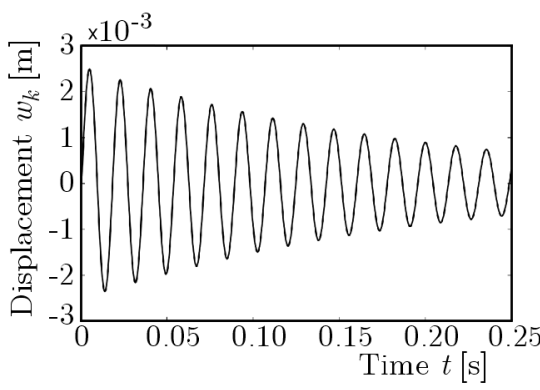

(b)

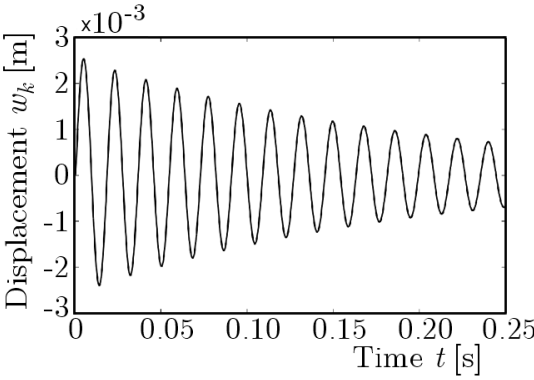

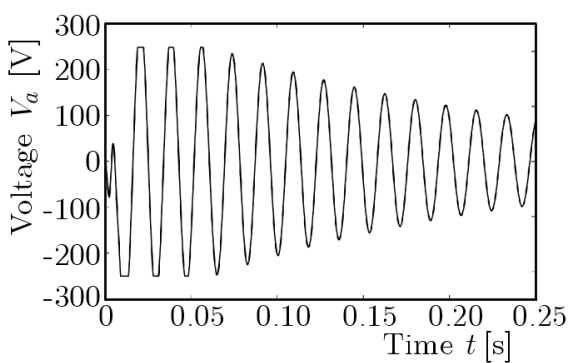

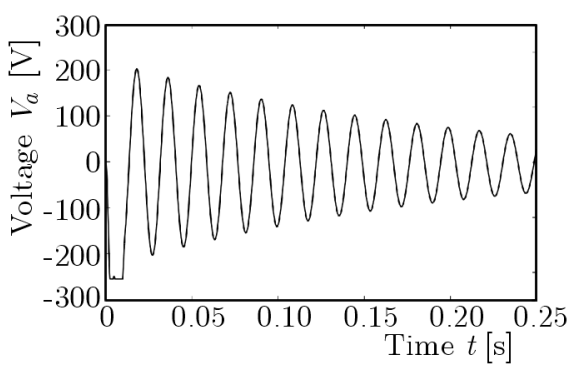

Fig. 4. Displacement of the free end of the beam $w_{k}$ and control voltage $V_{a}$ with step-wise change (fundamental mode); (a) $d=1$, (b) $d=0.1$

(a)

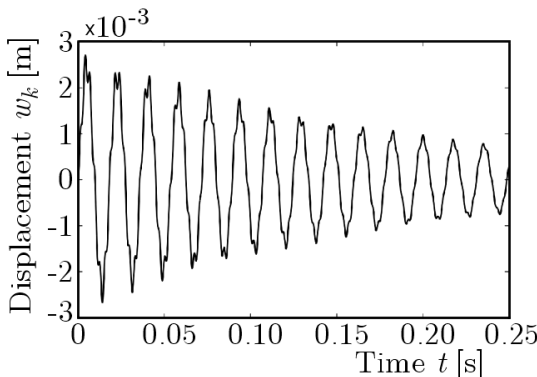

(b)

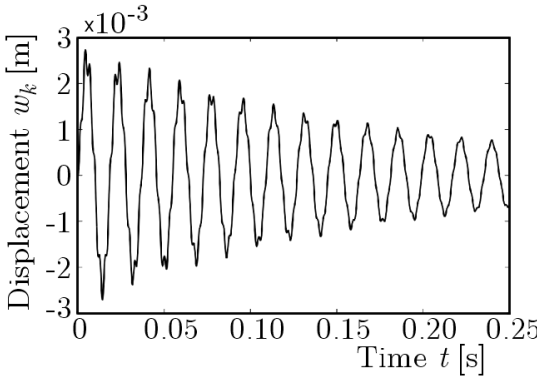

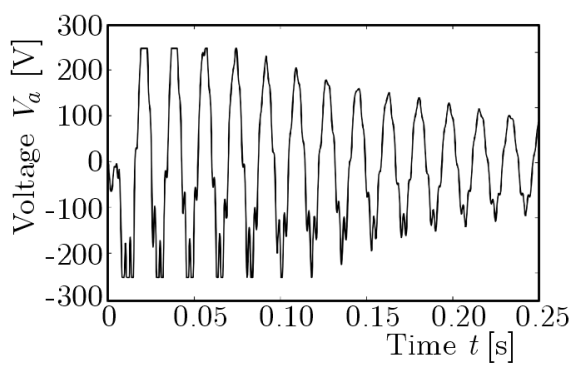

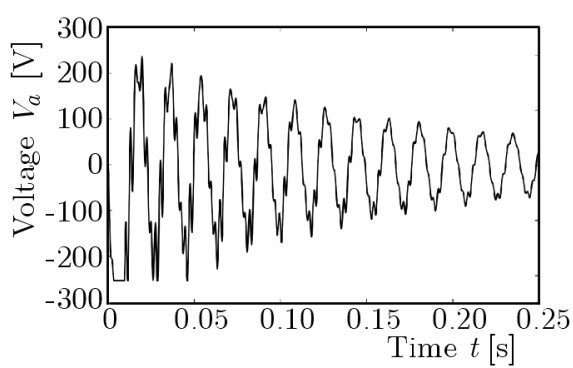

Fig. 5. Displacement of the free end of the beam $w_{k}$ and control voltage $V_{a}$ with step-wise change (two lowest modes); (a) $d=1$, (b) $d=0.1$

When applying this procedure, one should exercise caution, since when the value of $r$ is too high, the neural control system may become unstable and some additional measures to prevent this from happening would need to be taken.

\section{Summary}

The purpose of this paper is to present possible options for the active vibration damping of a clamped-free beam with piezoelectric patches using neural network control. Two control methods have been chosen and used for this purpose. Due to the complex nature of neural network control, several factors have to be considered. Several changes to the most important of these factors are 
presented in this study. It can be shown that by properly setting and adjusting these parameters the control can become faster and more effective.

Although the paper has been limited to the simulation studies, the results obtained and the techniques used are belived to be of help in the design of a neural controller of a physical smart beam.

\section{References}

1. Chor S., 2002, Vibration Control - Encyclopedia of Smart Materials, John Wiley \& Sons, New York

2. Crawley E., De Luis J., 1987, Use of piezoelectric actuators as elements of intelligent structures, AIAA Journal, 25, 1373-1385

3. Cupiat P., 2008, Coupled Electromechanical Vibration Problems for Piezoelectric DistributedParameter Systems, Politechnika Krakowska, Kraków

4. Flasiński M., 2011, Introduction to Neural Networks (in Polish), Wydawnictwo Naukowe PWN

5. Hagan M.T., Demuth H.B., 1999, Neural networks for control, Proceedings of the 1999 American Control Conference, San Diego, 1642-1656

6. Korbicz J., Obuchowicz A., Uciński D., 1994, Artificial Neural Networks (in Polish), Akademicka Oficyna Wydawnicza PLJ

7. Medsker L., JAin L.C., 1999, Recurrent Neural Networks: Design and Applications, CRC Press, $325-354$

8. Narendra K.S., Parthasarathy K., 1990, Identification and control of dynamical systems using neural networks, IEEE Transactions on Neural Networks, 1, 4-26

9. NizioŁ J., 1989, Principles of Machine Vibration (in Polish), Wydawnictwo PK, Kraków

10. Nørgaard M., Ravn O., Poulsen N,K., Hansen L.K., 2003, Neural Networks for Modelling and Control of Dynamic Systems, Springer

11. Omidvar O., Elliot D.L., Edit., 1997, Neural Systems for Control, Academic Press

12. Passino K.M., 2005, Biomimicry for Optimization, Control and Automation, Springer

13. Preumont A., 1997, Vibration Control of Active Structures, Kluwer Academic Publisher

14. QIU Z., Zhang X., Ye C., 2012, Vibration suppresion of a flexible piezoelectric beam using BP neural network controller, Acta Mechanica Solida Sinica, 25, 417-428

15. Rutkowski L., 2009, Methods and Algorithms of Artificial Inteligence (in Polish), Wydawnictwo Naukowe PWN

16. Smyser C.P., Chandrashekhara K., 1997, Robust vibration control of composite beams using piezoelectric devices and neural networks, Smart Materials and Structures, 6

17. Tylikowski A., 1993, Stabilization of beam parametric vibrations, Journal of Theoretical and Applied Mechanics, 31, 657-670

18. Valoor M.T., Chandrashekhara K., Agarwal S., 2001, Self-adaptive vibration control of smart composite beams using recurrent neural architecture, International Journal of Solids and Structures, 38, 7857-7874 